\title{
Long Period Fiber Grating for Biosensing: An Improved Design Methodology to Enhance Add-Layer Sensitivity
}

\author{
Sankhyabrata Bandyopadhyay, Ignacio Del Villar, Nandini Basumallick, Member IEEE, Palas Biswas, \\ Member IEEE, Tanoy Kumar Dey and Somnath Bandyopadhyay, Member IEEE
}

\begin{abstract}
We present our theoretical study on the design of LPFG sensor where its add-layer sensitivity is enhanced. addlayer sensitivity quantifies the sensitivity of the sensor to the changes taking place within few tens of nanometers around the receptor molecules. Two different methodologies: the use of dual overlay layer and tailoring of the intermodal separation between two cladding modes, have been used to enhance the add-layer sensitivity. Using coupled mode analysis we compute several examples to carry out a detailed comparative analysis between the results obtained, focusing on the cladding mode near mode transition.
\end{abstract}

Index Terms-Long period fiber grating, Couple mode theory, Cladding modes, add-layer sensing, Bio-sensing

\section{INTRODUCTION}

Long period fiber grating (LPFG) has played a major role in the domain of sensors for more than two decades [1]. A recently published review article and the cross references provide a clear idea about the state of the art [2], which shows that LPFG based refractive index (RI) sensors have immense potential in biological sensing applications. In LPFG sensors, the evanescent field of the cladding modes interacts with the surrounding medium and the resonant wavelength $\lambda_{\text {res }}$ of each cladding mode shifts due to the change in the surrounding refractive index $\left(\mathrm{n}_{\mathrm{SRI}}\right)$. The sensitivity $(\mathrm{S})$ is represented using the parameter $\mathrm{d} \lambda_{\text {res }} / \mathrm{dn}_{\mathrm{SRI}}$ and is defined in terms of nanometer per refractive index unit (nm/RIU).

It has already been established that SRI sensitivity of a cladding mode is significantly increased when it operates near the turn-around-point (TAP) or the so called dispersion turn around point (DTP) of the phase matching curve (PMC) [3]. Moreover, it can be enhanced even further if the initial

Manuscript received July 31, 2017;

The authors are with the CSIR-Central Glass \& Ceramics Research Institute, City-Kolkata, PIN-700032, India (e-mail: somnath@cgcri.res.in). Sankhyabrata Bandyopadhyay is a Ph. D. student of AcSIR, CGCRI, Kolkata. This work was supported by Council of Scientific Research (CSIR), India during the 12th Five Year Plan. Project Nos. ESC-0102 and ESC-0110.

I. Del Villar is with Public University of Navarra, 31006 Pamplona, Spain. (e-mail: ignacio.delvillar@unavarra.es). The author I. D. Villar thankfully acknowledge the support of the Spanish Agencia Estatal de Investigación (AEI) and Fondo Europeo de Desarrollo Regional (FEDER) (TEC201678047-R) and the Government of Navarre through the project with reference 2017/PI044 coupling condition of the cladding mode is tailored suitably [4]. Another important technique is to operate the cladding mode near mode transition (MT) [5,6]. The most interesting approach seems to be the design where a combined effect of working around DTP and MT is used [7,8]. These LPFG sensors, with excellent SRI sensitivity, are able to provide ample information about the dynamic interactions taking place around the surface during bio-molecular interactions [9-11].

It is important to note that standard biosensing schemes require deposition of several layers on the surface of the transducer. In general, there is a layer of polymeric material which is used as a biofunctional layer (BFL). A layer of biomolecules which acts as a receptor layer (RL) is then immobilized on the BFL. Finally, the trapping mechanism of the bioanalytes takes place in the form of attachment of another layer, say the analyte layer (AL), on the surface of the RL. All these mechanisms remain confined hardly within few tens to few hundreds of nanometers of the fiber surface. More importantly, to determine the biomolecular interaction that is taking place on the surface, a washing step in a buffer solution is necessary and the system is always kept immersed in the same buffer solution. In this situation, when LPFG sensor is used to quantify the binding interaction of analyte with the receptor molecule, it is therefore more important to estimate the wavelength shift due to change in thickness of the AL due to adsorption of analyte molecules on RL, rather than measuring the wavelength shift due to RI change of the bulk surrounding medium. The change in analyte layer thickness is certainly an important measurand because the adsorbed thickness is related to the concentration of the analyte solutions [12]. Also it has been observed [9-11] that wavelength shifts related to addition of thin layers are shorter compared to the change in RI of the bulk material surrounding the LPFG. Moreover, a given value of $\mathrm{d} \lambda_{\text {res }} / \mathrm{dn}_{\mathrm{SRI}}$ for a particular sensor cannot predict the thickness of the adhered analyte layer on the receptor molecule.This has never been addressed in the theoretical models used so far to design high performance LPFG based RI sensors.

In this paper, using coupled mode theory, we have theoretically computed the response of a cladding mode of an LPFG, considering multiple thin film overlays of finite thicknesses, as normally needed in biosensing, and calculated the resonant wavelength shift of a cladding mode either for a change of thickness or for the RI of the analyte layer. We propose to use the terminology 'add-layer Sensitivity' $\left(\mathrm{S}_{\mathrm{A}}\right)$ to quantify the wavelength shift of a cladding mode related to changes that are confined within the analyte layer only. We 


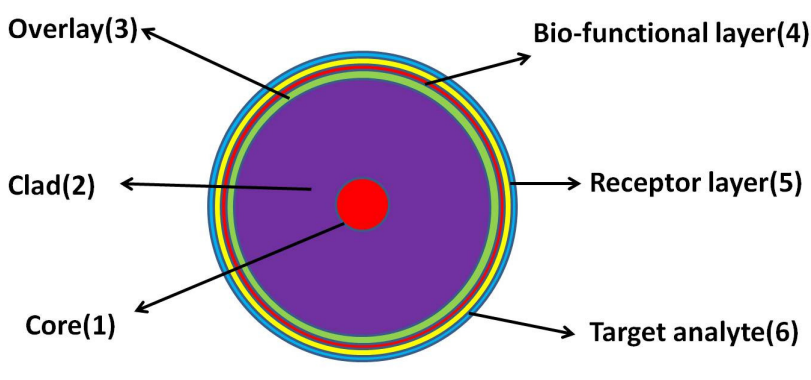

Surrounding medium (7)

Fig.1a.Optical fiber with multiple concentric layers as used in biosensing application

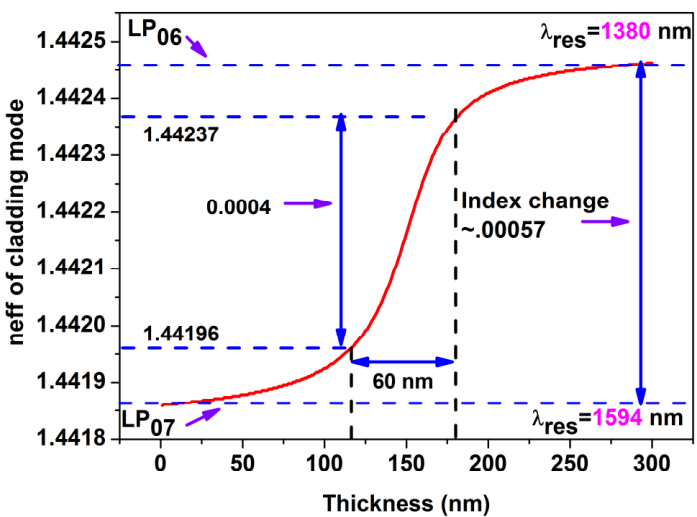

Fig.1b. Computed effective index of $\mathrm{LP}_{07}$ cladding mode as a function of $\mathrm{OL}$ thickness for SMF 28 fiber with BFL and RL

studied the sensitivity $\left(S_{A}\right)$ of a cladding mode near mode transition. To begin with, we numerically computed add-layer sensitivity of a cladding mode in a standard single mode fiber (SMF). Subsequently, based on the concept proposed in [13], we extended our analysis for a double clad fiber where we show that the sensitivity at MT can be enhanced almost by five to six times if the outer clad is tailored suitably. Finally, we investigated the effect of tuning the intermodal separation of the cladding modes in a standard SMF [14] on the add-layer sensitivity of the concerned cladding mode around MT. It has been shown that sensitivity can be enhanced by similar order of magnitude. A detailed discussion and several numerical examples analyzed by the proposed method are elaborated in the following sections.

\section{NUMERICAL RESULTS}

\section{A. Definition of the problem}

So far, to design an LPFG sensor at MT for high sensitive RI measurement, a thin film overlay (OL) having higher RI than the fiber cladding is deposited and its thickness is optimized so that the effective index $\left(\mathrm{n}_{\mathrm{eff}}\right)$ of the cladding mode of interest is positioned in the region of highest variation as a function of the OL thickness [5]. Secondly, a high RI material is preferred to accomplish a steep slope so that the resonant wavelength changes significantly as a function of the surrounding RI. To design a sensor as discussed above, computation of the propagation constants of the modes and coupled mode analysis is done typically for a four-layer fiber structure (i.e. core-clad-OL-surrounding medium). The sensitivity ( $\mathrm{S}$ ) of the sensor is generally obtained by recording the shift of the resonant wavelength of the cladding mode as a function of the surrounding RI (i.e. using the parameter $\mathrm{d} \lambda_{\text {res }} / \mathrm{dn}_{\text {SRI }}$. Conversely, Fig.1(a) shows a standard layered structure generally required on the fiber surface, to use an LPFG as a biosensor. As discussed in the previous section, on the OL, generally there is a polymeric layer which acts as a bio-functional layer (BFL). In addition to this, a receptor layer (RL) of biomolecules is immobilized on the BFL. The final layer is composed of the molecules of the target analyte (AL). It is apparent from Fig. 1(a) that the thickness of OL has to be optimized for specific BFL and RL, to position the cladding mode optimally at the high sensitive zone. Secondly, specifically for biosensing applications, the model should explicitly talk about the sensitivity with respect to the thickness of the added analyte layer and also the change in the $\mathrm{RI}$ of the layer of analytes. Therefore, the add-layer sensitivity $\mathrm{S}_{\mathrm{A}}$ may be computed as follows:

$$
S_{A}=\frac{d \lambda_{\text {res }}}{d h_{A L}}+\frac{d \lambda_{\text {res }}}{d n_{A L}}
$$

where the first term is the sensitivity of the cladding mode for a change in the layer thickness (h) of the analyte layer (say

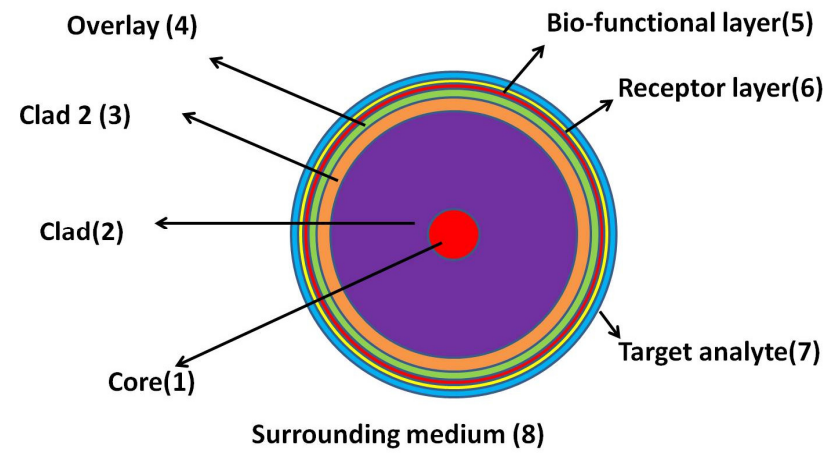

Fig.2. Layer architecture on an LPFG as considered for the DCF- 13 fiber.

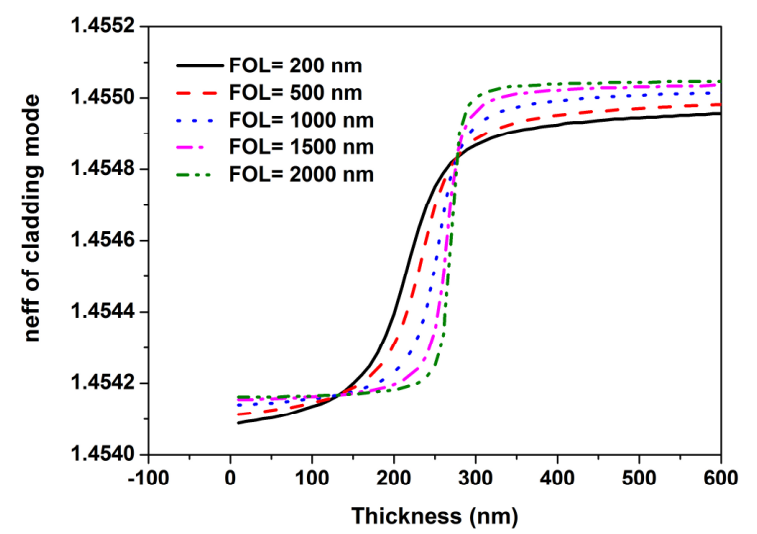

Fig.3. Effective index of $\mathrm{LP}_{07}$ cladding mode as a function of $\mathrm{OL}$ thickness for DCF-13 fiber computed for varying FOL thickness with a fixed BFL and RL. 


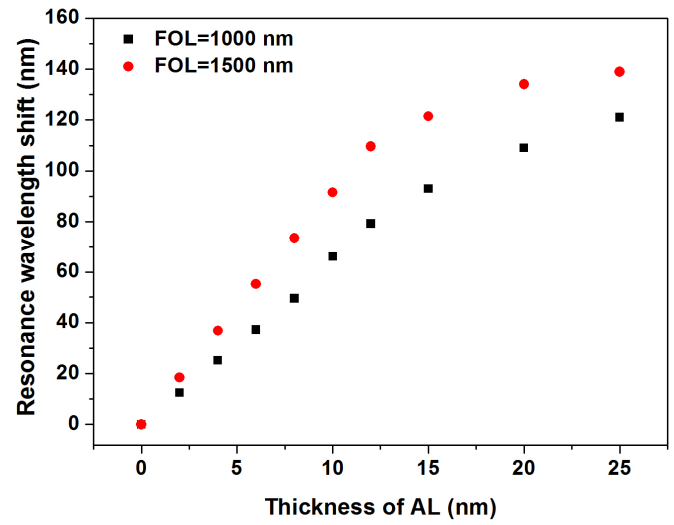

Fig.4. Shift of resonance wavelength of $\mathrm{LP}_{07}$ cladding mode of DCF-13 at maximum sensitive point with adhesion of AL.

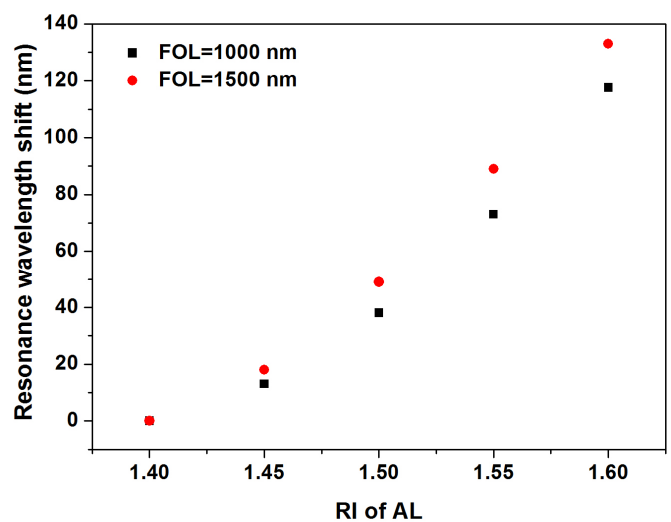

Fig.5. Computed shift of the resonant wavelength of $\mathrm{LP}_{07}$ cladding mode as a function of RI of the AL for the case of DCF-13 fiber.

$\mathrm{S}_{\mathrm{h}}$ ) and the second term is the sensitivity due to the changes in the layer refractive index (say $\mathrm{S}_{\mathrm{LRI}}$ ). Numerically simulated results are detailed in the following sections for different fiber structures as discussed in the previous section.

\section{B. Computation of Add-layer Sensitivity}

In our model we consider a seven layer waveguide structure (e.g. core-clad-OL-BFL-RL-AL-surrounding medium) as indicated in Fig. 1(a). We have pursued standard methodologies to compute the propagation constants of cladding modes for this multilayered structure and coupled mode equations were also solved for the same structure [15]. We considered fiber parameters for SMF-28 (Corning Inc.), BFL thickness of $5 \mathrm{~nm}$ with $\mathrm{RI}=1.54$ (e.g. Eudragit L-100 in general or may be polystyrene) and RL of thickness of $5 \mathrm{~nm}$ with $\mathrm{RI}=1.6$ (e.g. in general for dry protein). The RI of the OL is considered to be 1.7, which can be thought to be of a layer of Ti-doped $\mathrm{SiO} 2$ and may be deposited by sol-gel technique.

Fig. 1(b) shows the variation of the effective index of $\mathrm{LP}_{07}$ mode as a function of OL considering the specific BFL and RL mentioned above. From this plot a clear idea about the thickness of the OL for a specific functional and receptor layer is obtained. We computed the shift of resonant wavelength due to the addition of a layer of a bio-analyte with average RI of 1.55 with varying thickness. In general, the RI of bio-

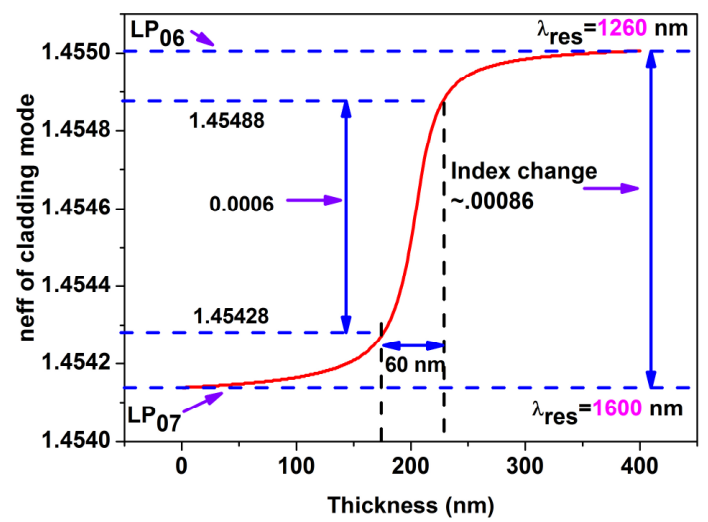

Fig.6a. Effective index of $\mathrm{LP}_{07}$ cladding mode as a function of OL for DCF13 fiber computed for an FOL of $1.5 \mu \mathrm{m}$ with BFL and RL on the OL

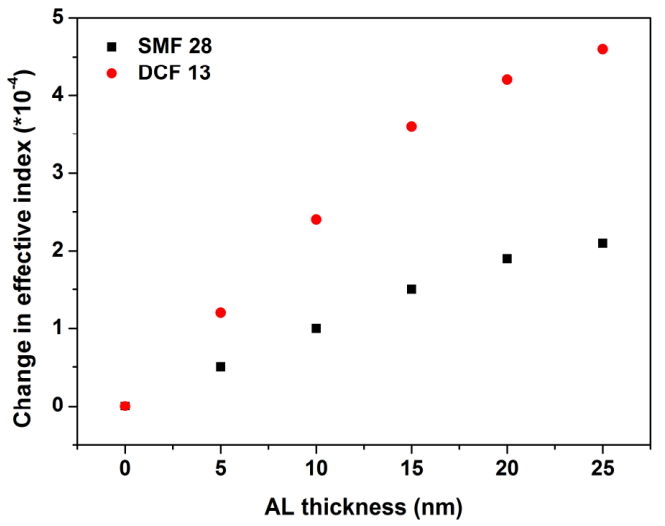

Fig.6b Change of effective index change of $\mathrm{LP}_{07}$ cladding mode for SMF 28 and DCF-13as a function of AL thickness.

analyte molecules have wide variation, which depends on concentration, nature of the analyte molecules etc. [16]. We considered a specific value for the sake of simulations only. In the linear region we obtained the $S_{\mathrm{H}}$ to be $\sim 2.2 \mathrm{~nm}$ per $\mathrm{nm}$ of AL layer. $\mathrm{S}_{\mathrm{LRI}}$ was found to be $\sim 80 \mathrm{~nm} / \mathrm{RIU}$ for this case. As a typical estimate, adhesion of a layer of $\sim 1 \mathrm{~nm}$ of analyte molecules roughly corresponds to about $5 \mu \mathrm{g} / \mathrm{ml}$ of $\mathrm{IgG}$ concentration [12]. Therefore, to bring down the limit of detection (LOD) for adsorbed molecules, $S_{\mathrm{H}}$ needs to be enhanced. For specific applications where measurement of surface RI is more important than the change in the layer thickness, a sensor with high $S_{\text {LRI }}$ will be more useful. It may be pointed out that the numbers obtained in this example may be optimized for other RI values of the OL. Also the other established concept of operating the cladding mode near DTP [7] may also be included to enhance the ad-layer sensitivity with the combined effects. We have however explored another alternative approach for the enhancement of add-layer sensitivity which is discussed in the next section.

C. Add-layer Sensitivity with dual overlay layer

It was shown earlier [13] that, if an overlay layer of low RI material with an optimal thickness (namely the first thin film overlay layer (FOL)) is used on the fiber cladding before deposition of the thin film overlay of high index material (i.e. 
TABLE I

COMPARISON OF SENSITIVITY OF LP 07 MODE OF SMF 28 AND DCF-13

\begin{tabular}{|c|c|c|c|c|c|c|}
\hline & \multicolumn{3}{|c|}{ Without FOL } & \multicolumn{3}{|c|}{ With FOL } \\
\hline $\begin{array}{c}\text { Overlay } \\
\text { RI }\end{array}$ & $\begin{array}{l}\text { Optimum OL } \\
\text { thickness in nm }\end{array}$ & $\begin{array}{c}\mathrm{S}_{\mathrm{H}} \\
(\mathrm{nm} / \mathrm{nm})\end{array}$ & $\begin{array}{c}\mathrm{S}_{\text {LRI }} \\
(\mathrm{nm} / \text { surface } \\
\text { RI) }\end{array}$ & $\begin{array}{c}\text { Optimum OL } \\
\text { thickness in nm } \\
\text { (with FOL }=1.5 \mu \mathrm{m} \text { ) }\end{array}$ & 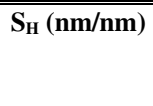 & $\begin{array}{c}\mathbf{S}_{\text {LRI }} \\
(\mathrm{nm} / \text { surface } \\
\text { RI) }\end{array}$ \\
\hline 1.52 & $\sim 430$ & $\sim 1.1$ & $\sim 30$ & $\sim 650$ & $\sim 7$ & $\sim 230$ \\
\hline 1.6 & $\sim 350$ & $\sim 1.6$ & $\sim 50$ & $\sim 540$ & $\sim 8.4$ & $\sim 340$ \\
\hline 1.7 & $\sim 150$ & $\sim 2.2$ & $\sim 80$ & $\sim 240$ & $\sim 9.3$ & $\sim 460$ \\
\hline 1.8 & $\sim 120$ & $\sim 2.8$ & $\sim 120$ & $\sim 170$ & $\sim 14$ & $\sim 580$ \\
\hline 1.9 & $\sim 85$ & $\sim 3.6$ & $\sim 180$ & $\sim 130$ & $\sim 17$ & $\sim 710$ \\
\hline 2 & $\sim 50$ & $\sim 4.7$ & $\sim 250$ & $\sim 100$ & $\sim 22.5$ & $\sim 850$ \\
\hline
\end{tabular}

OL), then the mode transition can be achieved with reasonably steeper slope. We have explored this concept using dual overlay layer and analysed its influence on $S_{\mathrm{H}}$ and $\mathrm{S}_{\mathrm{LRI}}$. In our simulation we considered a commercially available double clad fiber DCF-13 (Thorlabs Inc.). The RI of its outer clad (say clad2) is lower than its inner clad (clad1). Therefore, when an overlay layer of high index material is deposited on the outer clad (clad2), the outer clad can be considered as a first thin film overlay (FOL). Because the clad2 is the outer most layer of the fiber, it can be tailored as per the requirement during fabrication. Modified layered structure considered in this example is shown in Fig.2. In this case we considered an eight layer cylindrical waveguide structure (e.g. core-clad1-clad2(FOL)-OL-BFL-RL-AL-surrounding). As discussed above, clad2 acts as the FOL and its RI is lower than the RI of the second thin film overlay (OL). The fiber parameters of DCF-13, measured with an optical fiber analyser are: core radius $=4.5 \mu \mathrm{m}$, clad 1 radius $=52.5 \mu \mathrm{m}$, clad 2 thickness $=10 \mu \mathrm{m}, \quad \mathrm{RI}$ core $=1.4620, \quad \mathrm{RI}_{\text {clad } 1}=1.4571$ and RI $I_{\text {clad2}}=1.4434 @ 1550$ nm.

Using coupled mode analysis we find the influence of the thickness of the FOL on the transition of $\mathrm{LP}_{07}$ cladding mode to its next lower order. The other parameters for OL, BFL and $\mathrm{RL}$ were identical to those considered in the previous case for a standard SMF.

Fig. 3 shows the variation of $\mathrm{n}_{\mathrm{eff}}$ of $\mathrm{LP}_{07}$ cladding mode as a function of OL for different thicknesses of the FOL. At the outset, it becomes apparent that the concept [13] of using a dual overlay layer originally proposed for enhancing the bulk

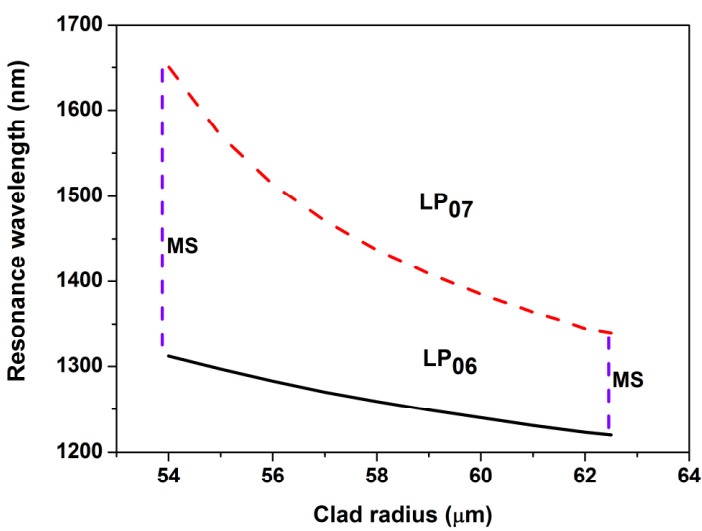

Fig.7 Computed shift of resonance wavelength of $\mathrm{LP}_{06}$ and $\mathrm{LP}_{07}$ cladding mode of SMF 28 fiber with reduction of clad diameter
RI sensitivity still holds good for a layered structure on the OL and can be useful to enhance the add-layer sensitivity. The slope of the MT curve gradually increases with increasing FOL for a fixed RI of the OL. In practice it is preferable to maintain FOL at lesser value than its maximum limit because positioning the operating point of the cladding mode in the linear part of the MT curve becomes extremely difficult in practice. It is important to note that the optimum FOL layer thickness as discussed above depends on many parameters like the RI values and the thicknesses of all the layers considered in the example. Therefore, the design of sensor certainly has to be application specific. Consequently, we compute the shift of the resonant wavelength as a function of AL thickness and also due to change in RI of the AL layer.

Fig.4 and Fig.5 show the computed results for two different FOL layer thicknesses. It should be pointed out that for an optimum FOL thickness, the RI of OL layer also contributes to sensitivity enhancement. For a better understanding we therefore, have computed $\mathrm{S}_{\mathrm{H}}$ and $\mathrm{S}_{\mathrm{LRI}}$ for $\mathrm{LP}_{07}$ cladding mode for different RI of the OL. We firstly consider the structure as shown in Fig. 1(a), where there is no FOL and then the structure as shown in Fig 2 with an FOL of 1500nm. Table I details the result. It is clear that for all the cases $S_{H}$ and also $\mathrm{S}_{\mathrm{LRI}}$ can be enhanced by five to six times when the cladding mode is designed to operate around the MT using a dual overlay layer. The sensitivity, i.e. wavelength shift of the resonant wavelength of $\mathrm{LP}_{07}$ cladding mode is enhanced to around $20 \mathrm{~nm}$ by adhesion of $1 \mathrm{~nm}$ of analyte molecules. Consequently, with an adequate wavelength detection

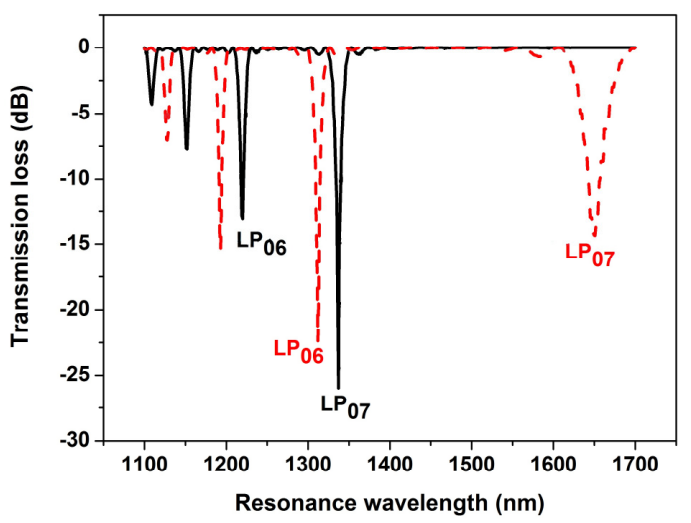

Fig.8. Simulated spectrum of LPFG1 with $62.5 \mu \mathrm{m}$ clad radius (black solid line) and $53 \mu \mathrm{m}$ clad radius (red dashed line) 




Fig.9. Computed spectrum of LPFG1 with clad radius $53 \mu \mathrm{m}$ (red dashed line) and LPFG2 with $62.5 \mu \mathrm{m}$ (blue dash-dot line)

mechanism, adsorption behaviour on the surface at subnanometer level is possible with the designed LPFG sensor. It may be observed that the high sensitivity is achieved at the expense of a relatively higher thickness of OL in addition to a mandatory FOL of a suitable thickness.

\section{Add-layer Sensitivity with tuned inter modal separation}

As a consequence of our investigation, as described in the previous section, we make out another possible methodology for enhancing add-layer sensitivity. The concept relies on the principle of broadening the difference of effective index between two adjacent resonant cladding modes for which the mode transition takes place during measurement. Fig. 6a shows a specific example for the DCF-13 fiber where an MT curve is computed for an OL layer with $\mathrm{RI}=1.7$ and for an FOL of thickness $1500 \mathrm{~nm}$. We compare the MT curve as shown in Fig. 6(a) with Fig. 1(b) where the MT curve was computed for a standard single mode fiber with an OL layer of identical RI but without any FOL. It may be observed that difference of effective index between $\mathrm{LP}_{07}$ and $\mathrm{LP}_{06}$ is considerably large in the case of DCF fiber as compared to the SMF-28, an effect that plays an important role during mode transition. As seen from the results in Fig. 1(b) and Fig. 6(a), for a particular OL material (here RI of OL $=1.7$ ) the change in effective index of the cladding mode per unit layer of the



Fig.10 Computed results showing the variation of effective index of $\mathrm{LP}_{07}$ cladding mode for LPFG2

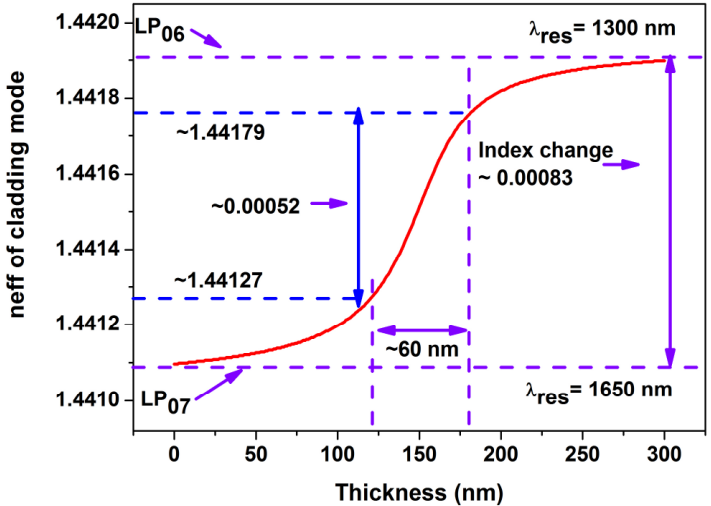

Fig.11. Variation of effective index of $\mathrm{LP}_{07}$ cladding mode as a function of OL for LPFG1 (radius $=53 \mu \mathrm{m})$

OL, while passing over the linear part of the MT curve, is always larger in the case of fibers having extended modal separation. Therefore, extending inter modal effective index is also a key attribute for getting a steeper linear zone during mode transition. Incorporation of an FOL of course adds to the effect. We computed the change of effective index of the $\mathrm{LP}_{07}$ mode as a function of $\mathrm{AL}$ and the results are shown in Fig. 6(b). The rate of change of effective index for DCF-13 fiber is irrefutably larger and so are the wavelength shift and the sensitivity.

The analysis of results obtained for DCF-13 fiber prompted us to explore the effect of tuning intermodal separation on the add-layer sensitivity. Tuning of the intermodal separation of the resonant cladding modes can be done simply by reducing the clad diameter [14] by etching. We computed shift of the resonant wavelength of $\mathrm{LP}_{07}$ and $\mathrm{LP}_{06}$ cladding mode as a function of clad radius for SMF-28 and the result is shown in Fig. 7. As an example we show the spectra of the resonant cladding modes of an LPFG (namely LPFG1) with a grating period $\Lambda=300 \mu \mathrm{m}$ in SMF-28 for clad radii $62.5 \mu \mathrm{m}$ and 53 $\mu \mathrm{m}$ in Fig. 8. We purposefully devised LPFG1 with a period of $300 \mu \mathrm{m}$ so that after reducing the clad the $\mathrm{LP}_{07}$ cladding mode is positioned around $1650 \mathrm{~nm}$ which in practice is a standard operating zone in $\mathrm{C}+\mathrm{L}$ telecommunication band where the optical sources are easily available. For

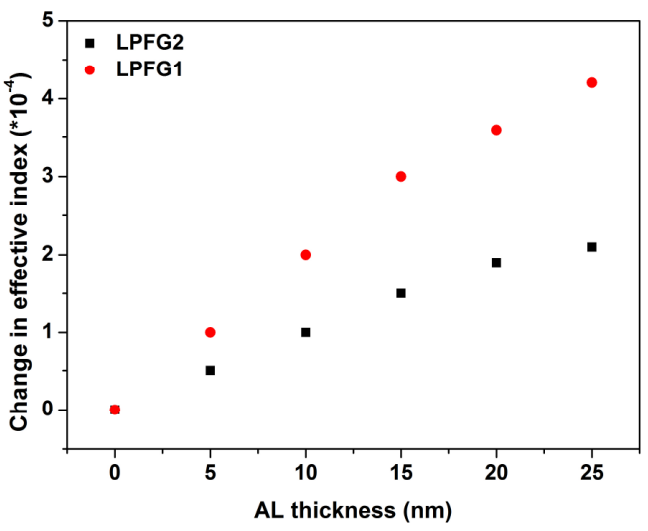

Fig.12. Computed results showing the variation of effective index of cladding mode of LPFG1 and LPFG2. 
TABLE II

SENSITIVITY OF LP 07 CLADDING MODE OF SMF 28 FIBER WITH ETCHING

\begin{tabular}{ccc}
\hline \hline Clad radius $(\boldsymbol{\mu m})$ & $\begin{array}{c}\mathbf{S}_{\mathbf{H}} \\
(\mathbf{n m} / \mathbf{n m})\end{array}$ & $\begin{array}{c}\mathbf{S}_{\mathbf{L R I}} \\
(\mathbf{n m} / \mathbf{L R I})\end{array}$ \\
\hline 62.5 & $\sim 2.2$ & $\sim 80$ \\
60 & $\sim 2.8$ & $\sim 110$ \\
58 & $\sim 3.7$ & $\sim 175$ \\
56 & $\sim 5$ & $\sim 290$ \\
54 & $\sim 8.1$ & $\sim 400$ \\
53 & $\sim 9.2$ & $\sim 685$
\end{tabular}

TABLE III

SENSITIVITY OF LP L7 $_{07}$ CLDDING MODE OF ETCHED FIBER (RAD $53 \mathrm{MM}$ ) WITH HIGHER VALUES OF OVERLAY RI

\begin{tabular}{ccc}
\hline \hline $\begin{array}{c}\text { Overlay } \\
\text { RI }\end{array}$ & $\begin{array}{c}\text { Required } \\
\text { OOT }(\mathbf{n m})\end{array}$ & $\begin{array}{c}\mathbf{S}_{\mathbf{H}} \\
(\mathbf{n m} / \mathbf{n m})\end{array}$ \\
\hline 1.7 & 150 & $\sim 9.2$ \\
1.8 & 120 & $\sim 11.5$ \\
1.9 & 85 & $\sim 14.4$ \\
2 & 50 & $\sim 18.5$
\end{tabular}

performance comparison, we simulate another LPFG (namely LPFG2) with $\Lambda=360 \mu \mathrm{m}$ with clad radius $=62.5 \mu \mathrm{m}$, so that the $\mathrm{LP}_{07}$ clad mode is also positioned around $1650 \mathrm{~nm}$. The spectra of LPFG1 (clad radius $=53 \mu \mathrm{m})$ and LPFG2 $(\mathrm{clad}$ radius $=62.5$ ), considered for our further analysis, are shown in Fig. 9. We subsequently computed the MT curve for $\mathrm{LP}_{07}$ cladding modes for LPFG1 and LPFG2 considering the same multilayered structure as given in Fig. 1(a) and the results are shown in Fig. 10 and Fig. 11 respectively. The figures are selfexplanatory and show that, in the linear zone of the MT curve, the change in effective index with respect to OL layer is larger in the case of LPFG1, which has larger intermodal separation as compared to LPFG2. Fig. 12 shows the variation of $n_{\text {eff }}$ for the $\mathrm{LP}_{07}$ mode as a function of the thickness of $\mathrm{AL}$ both for LPFG1 and LPFG2. The rate of change of effective index for the concerned cladding mode is larger for LPFG1 as predicted. We further progressed to compute $S_{H}$ and $S_{L R I}$ for different clad radius i.e. for different modal separation between $\mathrm{LP}_{07}$ and $\mathrm{LP}_{06}$ for $\mathrm{OL}$ layer $\mathrm{RI}=1.7$. The enhancement of sensitivity is encouraging and is almost similar to what we have achieved with a DCF-13 fiber with dual overlay layer and for the same OL layer RI. The results are tabulated in Table II. We also investigated the influence of the RI of the OL layer on the sensitivity. The results are presented in Table III. The results as summarized in Table II and Table III show that the design based on tuning of the intermodal separation has several practical advantages. Firstly, a low cost SMF-28 gives us the solution. Secondly, a precise FOL layer can be avoided. It has been observed an error of few micro meters drastically alters the sensitivity. Finally, to achieve a sensitivity of similar order, requirement of the thickness of OL layer is almost half as compared to that needed in the case of dual overlay layer architecture. It is understandable that overlay layer deposition with this level of precision is a tedious and also a costly affair. So minimizing the layer thickness is always preferable.

\section{CONCLUSION}

Studies on the enhancement of add-layer sensitivity of a cladding mode in LPFG sensor have been presented. We analyzed add-layer sensitivity of the cladding mode near mode transition considering two different enhancement methodologies. Firstly, an LPFG with dual overlay layer and then an LPFG with tuned intermodal separation. Detailed performance analysis shows that both the methodologies permit to increase the sensitivity of LPFG sensors to a point where it is possible to detect the sub-nanometer scale adhesion of bioanalytes on the surface of the LPFG.

Although available established techniques like quartz crystal microbalance dissipation (QCM-D) based techniques [12], surface plasmon resonance (SPR) [17,18], approaches based on Bloch surface wave [19] and also high precision ellipsometric measurements are able to quantify the binding interaction of bio-molecules on the surface and also the change of surface RI respectively, there has been little effort to obtain similar results for the case of LPFG sensors. The design methodology discussed in this paper certainly positions LPFG sensors as another platform in this biosensing area. The methodologies proposed are practically implementable with the available state of the art of LPFG fabrication and of highly accurate layer deposition on the surface of the LPFG.

\section{REFERENCES}

[1]. H. J. Patrick, A. D. Kersey, and F. Bucholtz, "Analysis of the response of long period fiber gratings to external index of refraction," J. Lightwave Technol., vol. 16 no. 9, pp. 1606-1612 Sept. 1998.

[2]. Francesco Chiavaioli, Carlos A. J. Gouveia, Pedro A. S. Jorge, and Francesco Baldini, "Towards a Uniform Metrological Assessment of Grating-Based Optical Fiber Sensors: From Refractometers to Biosensors," Biosensors., vol-7, no. 2, pp.23-29, June. 2017.

[3]. X. Shu, L. Zhang, and I. Bennion, "Sensitivity Characteristics of Long-Period Fiber Grating," J. Lightwave Technol., vol. 20, no. 2, pp. 255-266, Feb. 2002.

[4]. P. Biswas, N. Basumallick, S. Bandyopadhyay, K. Dasgupta, A. Ghosh, and S. Bandopadhyay, "Sensitivity enhancement of turn around point long period gratings by tuning initial coupling condition", IEEE Sens. J., vol. 15, no.2, pp. 1240-1245, Feb. 2015.

[5]. I. Del Villar, I. R. Matias, F. J. Arregui and P. Lalanne, "Optimisatiom of sensitivity in long period fiber gratings with overlay deposition", Opt. Exp., vol. 13, no. 1, pp.5669, Dec. 2005.

[6]. P. Pilla, C. Trono, F. Baldini, F. Chiavaioli, M. Giordano and A. Cusano, "Giant sensitivity of long period gratings in transition mode near the dispersion turning point: an integrated design approach", Opt. Lett., vol. 37, no.19, pp. 4152-4154, Oct. 2012. 
[7]. I. Del Villar, "Ultrahigh-sensitivity sensors based on thinfilm coated long period gratings with reduced diameter, in transition mode and near the dispersion turning point", $O p t$. Exp., vol. 23, no.7, pp.8389-8398, Apr. 2015.

[8]. M. Smietana, M. Koba, P. Mikulic, and W. J. Bock, "Combined Plasma-Based Fiber Etching and DiamondLike Carbon Nano-overlay Deposition for Enhancing Sensitivity of Long-Period Gratings", J. Lightwave Technol., vol. 34, no. 19, pp.4615-4619, Oct. 2016.

[9]. P. Pilla, V. Malachovská, A. Borriello, A. Buosciolo, M. Giordano, L. Ambrosio, A.Cutolo, A. Cusano, "Transition mode long period grating biosensor with functional multilayer coatings", Opt. Exp., vol. 19, no.2, pp. 512-526, Jan. 2011.

[10].M. Smietana, M. Koba, E. Brzozowska, K. Krogulski, J. Nakonieczny, L. Wachnicki, P. Mikulic, M. Godlewski, and W.J. Bock, "Label-free sensitivity of long-period gratings enhanced by atomic layer deposited $\mathrm{TiO} 2$ nanooverlays", Opt. Exp., vol. 23, no.7, pp. 8441-8453, Apr. 2015.

[11].F. Chiavaioli, P. Biswas, C. Trono, S. Jana, S. Bandyopadhyay, N. Basumallick, A. Giannetti, S. Tombelli, S. Bera, A. Mallick, F. Baldini "Sol-Gel-Based Titania-Silica Thin Film Overlay for Long Period Fiber Grating-Based Biosensors" Anal. Chem., vol 87, no. 24, pp. 12024-12031, Nov. 2015.

[12].Cheng Zhou, Jean-Michel Friedt, Angelina Angelova,Kang-HoonChoi, Wim Laureyn, Filip Frederix, Laurent A. Francis, Andrew Campitelli, Yves Engel borghs, and Gustaaf Borghs "Human Immunoglobulin Adsorption Investigated by Meansof Quartz Crystal Microbalance Dissipation, Atomic Force Microscopy, Surface Acoustic Wave, and Surface Plasmon Resonance Techniques" Langmuir., vol.20, no.14, pp.5870-5878, 2004.

[13].Del Villar, I. R. Matias and F. J. Arregui, "Deposition of coatings on long period fiber gratings: tunnel effect analogy," Optical and Quantum Electron., vol. 38, pp. 655665, June. 2006.

[14].Sungchul Kim, Yoonchan Jeong, Seungwoo Kim, Jaejoong Kwon, Namki Park, and Byoungho Lee, "Control of the characteristics of a long-period grating by cladding etching", Appl. Opt., vol. 39, no. 13, pp. 2038-42, May 2000.

[15].E. Anemogiannis, E. N. Glytsis, and T. K. Gaylord," Transmission Characteristics of Long-Period Fiber Gratings Having Arbitrar Azimuthal/Radial Refractive Index Variations," J. Lightw. Technol., vol. 21, pp. 218227, Jan. 2003.

[16].N.J.Geddes, A.S.Martin , F.Caruso, R.S. Urquhart, D.N.Furlong, J.R.Sambles, K.A.Than, J.A.Edger "Immobilisation of IgG onto gold surfaces and its interaction with anti-IgG studied by surface plasmon resonance" Journal of Immunological Methods, vol.175, pp. 149-160. 1994.

[17].A. Ahluwalia, D. De Rossi, C. Ristori, A. Schiron and G. Serraa "A comparative study of protein immobilization techniques for optical immunosensors" Biosens. \& Bioelectr., vol. 7, pp. 207-214. June. 1991

[18].P. Kvasnicka and J. Homola" Optical sensors based on spectroscopy of localized surface plasmons on metallic nanoparticles: Sensitivity considerations" Biointerphases., vol. 3 no. 3, pp FD4 - FD11. Sept. 2008.

[19].Alberto Sinibaldi, Norbert Danz, Aleksei Anopchenko, Peter Munzert, Stefan Schmieder, Rona Chandrawati, Riccardo Rizzo, Subinoy Rana, Frank Sonntag, Agostino Occhicone, Lucia Napione, Simone De Panfilis, Molly M. Stevens, and Francesco Michelotti "Label-Free Detection of Tumor Angiogenesis Biomarker Angiopoietin 2 Using Bloch Surface Waves on One Dimensional Photonic Crystals" J. Lightwave Technol., vol. 33, no. 16, pp.33853393, Aug. 2015. 